\title{
LAS TRANSFORMACIONES EN LA EDUCACIÓN FORMAL DE LOS JOVENES ESTUDIANTES FRENTE AL MUNDO DEL TRABAJO: UN ESTUDIO COMPARATIVO DE LA POLÍTICA DE EDUCACIÓN EN BRASIL Y EN VENEZUELA
}

\author{
Manoel Montanha Soares ${ }^{1}$
}

\section{RESUMEN}

Este artículo tiene como objetivo analizar los cambios que se han procesado en los sistemas educativos de la segunda mitad del siglo XX en América Latina. Estos cambios son el resultado de cambios importantes en los sistemas de producción en todo el mundo. Después de la Segunda Guerra Mundial, los países de economía de centro reorganizaron sus economías y profundizaron la ideología de sus sistemas económicos se transfieren a los países del Tercer Mundo considerados. La población económicamente activa que se ha enfrentado a nuevos desafíos se compone de jóvenes de entre 15 y 24 años, que tienen aproximadamente el doble de la tasa de desempleo de los adultos. El resultado del estudio muestra una paradoja: la lucha por la igualdad y los derechos universales tradición reatualizam de ciudadanía restringida, propia de los países en desarrollo, asentado en la lógica de los privilegios que se extienden y no en los derechos universales.

Palabras clave: Joven mundo laboral, Brasil, Venezuela, desigualdad

\section{RESUMO}

Este artigo tem como objetivo analisar as mudanças que se processaram dentro dos sistemas de ensino a partir da metade do século XX na América Latina. Estas mudanças são produto das grandes transformações nos sistemas produtivos em escala mundial. Após a Segunda Guerra Mundial os países de economia central reorganizaram suas economias e aprofundaram a ideologia dos seus sistemas econômicos transferindo-as aos países considerados de Terceiro Mundo. A população economicamente ativa que mais tem enfrentado desafios é a composta por jovens entre 15 e 24 anos, que apresentam aproximadamente o dobro da taxa de desemprego dos adultos. $\mathrm{O}$ resultado do estudo mostra um paradoxo: a luta pela a igualdade e a universalização de direitos reatualizam a tradição de uma cidadania restrita, típica de países em desenvolvimento, assentada na lógica da expansão de privilégios e não na universalização de direitos.

Palavras-chave: Jovem; Mundo do Trabalho; Brasil; Venezuela; Desigualdade

\begin{abstract}
This article aims to analyze the changes that were processed within the education systems from the mid-twentieth century in Latin America. These changes are the result of major changes in production systems worldwide. After the Second World War, the countries of central economy reorganized their economies and deepened the ideology of their economic systems transferring them to Third World countries considered. The economically active population that has faced more challenges is composed of young people between 15 and 24 years, which have approximately twice the unemployment rate of adults. The result of the study shows a paradox: the struggle for equality and universal rights reatualizam tradition of citizenship restricted, typical of developing countries, seated in the logic of extending privileges and not on universal rights.
\end{abstract}

Keywords: Young Working World, Brazil, Venezuela, Inequality 


\section{Introducción}

Por qué las modernas formas de pensar la libertad, el orden moral y política privilegian el peso del educativo? No hay duda que ese enfasis no es gratuita tampoco neutra. Detrás de esa relación, siempre defendida en la construción del nuevo orden, hay una concepción del social y de su constituición, hay ideas e ideales políticos que no pueden ser ignorados, hay concepciones y compromisos con el orden social y económica, con el Estado y con un modelo de ciudadania para las clases inferiores.

Paolo Nosela, Educación y ciudadania

Los cambios que se procesaron en los sistemas de ensino a partir de la mitad del siglo XX son producto de las grandes transformaciones en los sistemas productivos en nivel mundial. Al final de la Segunda Guerra Mundial, los países de economia central reorganizaron sus economias y profundizaron la ideologia de sus sistemas económicos transferindolas a los países considerados de Tercer Mundo. Los sistemas educacionales fueron fuertemente alcanzados y la América Latina no ha quedado inmune al paradigma de la educación como un factor de desenvolvimento económico. Las políticas sociales de educación sufren directamente con la reestructuración productiva y las transformaciones tecnológicas en el mundo del trabalho.

Comprender el proceso de transformación en el mundo del trabajo y sus efectos sobre las estructuras de empleo y del trabajo llevanos directamente a la construcción de las políticas sociales de educación, teniendo en vista que trabajo y educación son un binómio constituyente de las estructuras generadoras de este modelo de sociedad y de las relaciones sociales por ella producidas. En los últimos treinta años, el mundo del trabajo ha pasado por transformaciones significativas que, en el limite, podemos afirmar alimentaron el proceso de precarización que tomaron proporciones significativas en los años 90 del siglo pasado y que avanzan en el siglo XXI. Este estudio se apoyará en un análisis interpretativa, fundamentada en un referencial metodológico crítico, sobre las acciones gobernamentales - las políticas públicas sociales de educación - formuladas e implementadas por los gobiernos de estes países visando responder a las demandas sociales articuladas en la esfera de la educación de jovenes estudiantes y el mundo del trabajo.

\section{La Consolidación del Estado en América latina y la formación de los Sistemas Públicos de Ensino: una tradición totalitária}

La formación de los sistemas públicos de ensino en América Latina se originan principalmente a través de los procesos de independencia que se presentaron a partir del siglo XIX de forma que

sufrieron la influencia directa de la Revolución Francesa y los ideales liberales que se irradiaron sobre América Latina sob diversos matizes, no se traduciendo necesariamente en imagen y semejanza al proceso promovido por los burgueses europeos, visto que la tradición histórica y cultural latino americana era cargada de un legado colonialista y totalitário. (SOLANO, 2001, p.06) 
La proclamación de ciudadanos iguales era un discurso profundamente asimétrico dentro de la lógica esclavista, no que refierese principalmente a la población indígena, negra e mestiza. Visto que la población latina americana en su gran mayoria era compuesta por índios, negros y mestizos que aun cargaban el onus de un legado histórico del pasado que permanecia vivo en los discursos literários de periodistas, cronistas, médicos, juristas e sanitaristas, llenos con el cientificismo positivista de la época que marcaban a hierro y fuego todos los no blancos (TELLES, 2006). De esta manera, estaba hecho el escenario de una América Latina que se queria desarrollada, pero, en verdad, presa a las corrientes culturales del colonialismo y del eugenesia eurocentrica del pasado y al peso del racismo, lanzando esta parcela de la población en condiciones inhumanas, en que las reglas de civilidad y moral burguesas eran francamente negadas.

A partir de la conformación histórica del contexto latino americano y sus fundamentos culturales importados es que la clase trabajadora establece, en su horizonte de luchas, la educación pública universalizada como medio de libertación de las pesadas corrientes del pasado colonialista. La lucha por participación ciudadana y acceso a los medios sociales y culturales que hasta entonces eran ofrecidos solamente a una élite blanca minoritária se presentava como pilar de las luchas operárias abriendo la posibilidad concreta de conquistálas a través de una educación pública de calidad.

\begin{abstract}
Al mismo tiempo, la educación que era ofrecida tenía como objetivo principal proporcionar una "cultura letrada" atada al sistema productivo ascendente. No era de hecho una educación nacional volvida para la construcción de una sociedad latina americana libre y igualitária, pues cargava en si organicamente una sociedad de casta y clases sociales bien definidas. (ARANHA, 1996, p.36)
\end{abstract}

Los princípios de igualdad liberal apuntaron para una concepción de sistema escolar público y universal donde todos los ciudadanos tendrían derecho de acceso y cuya calidad es garantizada por el Estado, e esto sucederia a través de la organización de un sistema nacional de educación. Este es un momento de gran importancia en la organización de los sistemas públicos de educación pues eses se tornaron base para la formación y consolidación de los nuevos Estados latino americanos. Así, es posible identificar la educación pública, a través del instruccionismo del siglo XIX como vocero del conocimiento de derechos y deveres individuales, así como la transmisión de nuevos valores contribuyendo para la creación de una nueva conciencia nacional.

El nacionalismo no fue solo una forma de afirmación versus las antiguas organizaciones coloniales, fue también marco de la definición de las fronteras y de la soberania. En los fines del siglo XIX, la integración en la vida nacional de inmigrantes estranjeros que han llegado en gran número para algunos países en América Latina fue sin duda uno de los objetivos delineados por los nuevos sistemas políticos. De este modo, sistemas escolares tornaronse agentes para la divulgación de valores nacionales, que deberían contribuir para la integración de la sociedad sobre un imaginário cultural comun. (GARCÍA, et al. 2001, p.31)

Surge entonces la preocupación con la organización de los sistemas nacionales de ensino en los países latino americanos. A partir de esta nueva organización nacional de los Estados, la cuestión del discurso político se vuelve para el público, y esto está presente en el texto constitucional y en proyectos para reforma y modernización de nuevos estados nacionales. Esta preocupación es una constante que aparece después de la independencia 
de las colonias, a pesar de este proceso estar lleno de instabilidades políticas que caracterizaron todo el proceso para la consolidación de las nuevas formas políticas en América Latina.

A partir de las transformaciones ocurridas en los estados latino americanos surgen las primeras intentativas de organizar un sistema nacional público de educación que se ha basado eminentemente en el ensino primário o elementar. Para los municípios fueron atribuídas, de acordo con la tradición colonial, el poder para mantener las escuelas públicas, así como nombrar y mantener profesores.

Sin embargo, a pesar de la prioridad atribuída a la diseminación de la educación básica en el discurso político de la época, y a pesar de las muchas intentativas de organizar y promover este nivel de ensino público es también evidente la preocupación y interés de creacción de nuevos liderazgos que formen un nuevo sistema de formación para las élites.

De esta maneira, los recursos presupuestarios volvidos para instituiciones públicas de nivel médio, que fueron delineando el nivel de ensino secundário, especialmente en la reforma o creación de universidades públicas federales recebian recursos directo del gobierno federal donde el Estado ejercia un monopólio haciendo del ensino superior un centro de formación de las élites blancas con una predominancia en la abocacia, en cuanto eso el ensino elementar quedava a merced de los pocos recursos de los municípios. (FERRER, et al. 2002, p. 75)

La instabilidad política ha caracterizado toda la primera y gran parte de la segunda mitad del siglo XIX en América latina, impediendo la organización de un sistema nacional de educación pública, reforzando la idea que la "independencia" de las colonias atendian también a los intereses del capitalismo europeo ascendente. Organizar los sistemas nacionales de educación pública significaba enfrentar la falta de recursos económicos, visto que la prioridad de la educación se volvia en gran parte en atender los niveles más altos de educación donde apenas una minoria tenía acceso y el arcaísmo del sistema fiscal y la precária naturaleza de los fondos municipales no conseguieron mantener los planes para una educación pública.

Esta situación comenzó a cambiar en el último tercio del siglo XIX, después de la definición de que la economia latino americana asumiria su papel como una exportadora de materias-primas para el mercado internacional, y con eso su impacto positivo no solo sobre las recetas del Estado pero traendo también incrementos para la estabilidad política e institucional. Con efecto, la consolidación de los Estados-nación ha traído consigo el fortalecimiento de grupos de interés muy grande, la burguesia nacional oriunda principalmente del modelo agrário tornase un enlace en la unificación de los Estados nacionales y juntamente con este sector de la sociedad viene la imposición y defesa de los intereses de estes grupos. (FERRER, et al. 2002, p. 98)

La construcción de los Estados nacionales trae la marca indeleble de los intereses de la burguesia ascendente latino americana. Un ejemplo de eso es la formación de un ejército nacional de caráter caudillesco y la construcción de un sistema jurídico con códigos civiles y penales que garantizasen la propriedad privada. Estos y outros factores fueron decisivos en la formación del aparato estatal. De esta forma, la vida política en este período fue caracterizada por una reducida representación popular, porque la política fue constituída solamente por la clase dirigente. En este período fueron emprendidas la 
modernización del Estado, incluyendo el desenvolvimento y proceso de formación de los sistemas nacionales de educación pública.

En general, la educación primária fue asumida como responsabilidad de los municípios, no solo en términos de financiamiento, pero también en muchos aspectos de la organización y gestión del sistema escolar. Este proceso de centralización se encaja en el proceso de consolidación del referido Estado, que se percibe como única forma de obtner las condiciones para la estabilidad de la sociedad elo progreso y el orden institucional. Mismo en los países que establecieron un sistema federal (México, Argentina, Venezuela), el gobierno central tenía un espacio bastante reservado en que se referia al domínio en la educación pública. Los gobiernos regionales asumen cada vez más responsabilidades en esta área.

En general, la centralización política es realizada en diferentes tasas de cada país, de acuerdo con sus características. Conflictos entre grupos en el domínio de determinadas regiones establecen caminos y intereses distintos para la educación pública. El poder de la Iglesia Católica, las oportunidades asociadas al mercado internacional, la importancia de la población de ex-esclavos, indígenas y las barreras a su propia sociedad "nacional" son factores determinantes en la organización de los sistemas públicos de educación. Nuevos régimenes liberales, el influjo de la imigración europea entre otros, fueron algunos de los fenómenos que fueron presentados en grados diferentes en cada país y moldaron su estabilidad política y social y ayudaron a construir un modelo de Estado nacional. (BENÍTEZ, 2009, p.14)

Percibise que en algun momento, todos los países latino-americanos, estableceron una estructura básica de sus sistemas escolares, cuya duración, a pesar de algunas reformas posteriores, permanecieron practicamente inalterados por lo menos hasta la Segunda Guerra Mundial.

La estructura de los sistemas escolares fue consagrada en la ley general del ensino, que, después de muchas vicisitudes de la primera mitade del siglo, ha ganado alguna estabilidad para el final del XIX e início del siglo XX. Estas leyes organizaron especialmente ensino básico o primário, sob el princípio comun en matéria de educación, que crea un órgano único de educación estructurado en el ensino primário, secundário y superior. Fue asi asegurada formalmente la igualdad de oportunidades educativas para que la mayoria de la población pudiese ingresar en el ensino primário gratuito y obligatório, asumiendo el Estado el papel de organizador del sistema escolar.

\section{JuVEntud, POBReza y desempleo en América latina: los desafios DEL MUNDO DEL TRABAJO}

El desempleo2 y el bajo número de los jovenes con empleo de 15 a 24 años en América Latina han contribuído para el aumento de la vulnerabilidad social de este segmento de la población, que en algunos países, amenaza la propia estabilidad social. Lidiar con este problema de forma estructural requiere un análisis del desempleo juvenil y de las opciones de políticas de educación y de programas gobernamentales para disminuir el desempleo y aumentar oportunidades de empleo y de integración de los jovenes en la sociedad. En los últimos años, el índice de desempleo abierto ha variado entre 8 y $12 \%$ en 
los países de América Latina según la OIT en un estudio denominado Panorama Laboral da América Latina y Caribe de 2011.

Aun de acuerdo con el informe ${ }^{3}$ de la OIT, denominado "Trabajo decente y juventud: América Latina" de 2011, hay 106 millones de jovenes entre 15 y 24 años y, de estes, 58 millones hacen parte de la fuerza de trabajo, de los cuales 10 millones están desempleados y 30 millones trabajan en economia informal. De los que trabajan, 63,7\% son hombres y 36,3\% mujeres. Otros 48 milhões están inactivos, o sea, no tienen, ni tampoco buscan empleo. Estimase que 22 millones de estes jovenes no estudian y tampoco trabajan, es decir, están "separados" institucionalmente y no tienen una actividad clara. Este número incluye 6 millones de desempleados que tampouco estudian, pero también abarca 16 millones de jovenes que no estudian ni están buscando trabajo y $81 \%$ de ellos, 22 millones de jovenes, viven en las ciudades y $72 \%$ son mujeres.

Del total de la población de jovenes, 49 millones estudian. De estes, 13 millones estudian y trabajan (58,2\% son hombres y $41,8 \%$ mujeres), 4 millones estudian y buscan, pero no consiguen trabajo y 32 millones solamente estudian (de los cuales 46,2\% son hombres y 53,8\% mujeres).

En América Latina las tasas de desempleo abierto oculta la realidad, en la medida en que la falta de oportunidades de empleo hace aumentar el llamado desempleo "oculto", donde las personas quedan desempleadas, procuram y no encuentran empleo, dejan de procurar y asi acabam saliendo del universo captado por los indicadores de desempleo. Por ese motivo, la tasa de desempleo abierto, todavia útil como indicador de aumentos o decréscimos a lo largo del tiempo, no refleja adecuadamente el cuadro total de desempleo abierto y disimulado (subempleo) y tampoco el cuadro de la pobreza.

En esta línea de abordaje el caso de la juventud en América Latina es más grave aun, las tasas de desempleo juvenil han crecido acentuadamente entre 2000 y 2010. Competieron para eso la inserción de las economias desenvolvidas en el mercado internacional más competitivo, el proceso de reestructuración económica y de privatizaciones sobre los países de economia emergente, así como los cambios tecnológicos y organizacionales que, a su vez, han cambiado la estructura ocupacional y los perfiles de profesionales de los trabajadores. Es importante decir que el desempleo juvenil en los últimos años creció más que el desempleo general. Veamos el cuadro abajo:

En la mayoria de los países de América Latina, las tasas de desempleo abierto entre los jovenes de 15 a 24 años son aproximadamente el doble de las tasas de los adultos. En Brasil, la tasa de desempleo, en general subió de 3\% en 1989 para más de $8 \%$ en 1997. En 2002, esa tasa sobe hasta $10 \%$, alcanzando $11,6 \%$ entre la población economicamente ativa urbana. Para el grupo de personas con edad entre 10 y 24 años, la tasa casi triplicó: pasó de cerca de 5\% en 1989 para más de 14\% en 2000. En 2009/2010 alcanzó cerca de 19\%, con variaciones significativas entre diversos grupos etarios. En el tercer trimestre de 2010 el

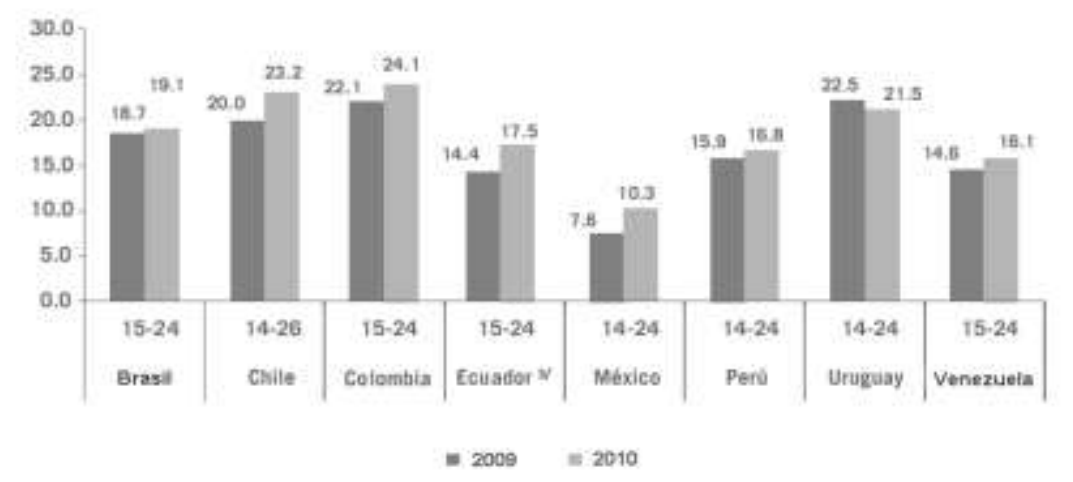

América Latina y el Caribe ( 8 paises): Tasa de desempleo urbano juvenil. I trimestre - III trimestre 2009 y 2010 (porcentajes)

Fuente: OाT sabir ta tase de informacitar oficial de las ancuestas do hogares on los onites 
desempleo entre los jovenes brasileros de 15 a 24 años ha variado de 17,8\%, para aquellos con edad entre 15 y 17 anos, en 13,3\%, para el grupo de 18 a 24 años. Eso demuestra que la tasa de desempleo de los jovenes es tres veces mayor de que la tasa de desempleo del grupo con edad acima de 25 años según el mismo informe "Panorama Laboral da América

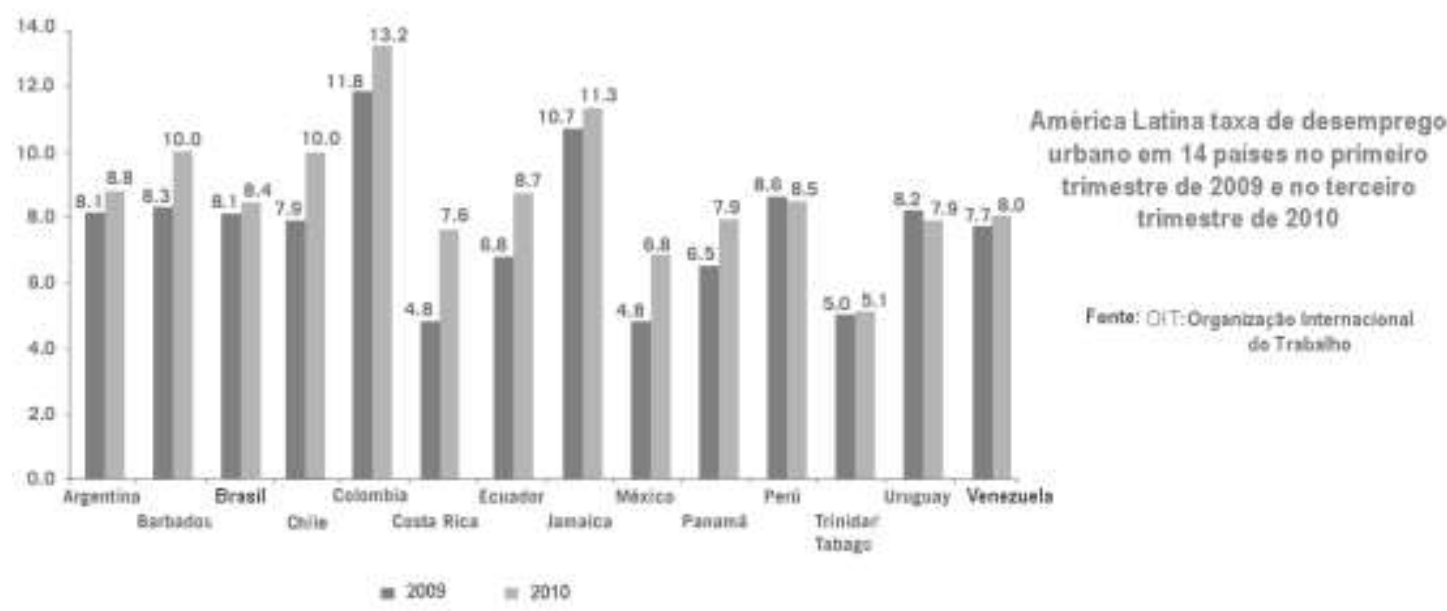

Latina y Caribe de 2010 de la OIT”. Veamos el gráfico abajo:

Entre los jovenes en América Latina, las tasas de desempleo de las mujeres jovenes son también, con frecuencia, más elevadas que las de los hombres jovenes. Las tasas de los jovenes con necesidades educacionales especiales, las de los jovenes provenientes de minorias étnicas y las de los jovenes de bajo nivel de educación son aun bien mayores. Entre las mujeres jovenes con edad entre 18 y 24 años, en 2009, el desempleo ha llegado a 17,1\%. En el grupo de 15 a 17 años, la tasa correspondiente fue de 22,3\%, representando, respectivamente, 7 y 4 puntos percentuales acima de los hombres jovenes en esas mismas grupos etarios.

Abajo la tabla evaluativa de la OIT sobre tasa percentual de empleo y desempleo en Brasil y en Venezuela entre 2010 y 2011:

\begin{tabular}{|l|l|l|l|l|}
\hline Países & $\begin{array}{l}\text { Tasa de } \\
\text { empleados } \\
(\mathbf{2 0 1 0}) \mathbf{- 1 5 - 2 4} \\
\text { anos }\end{array}$ & $\begin{array}{l}\text { Tasa de } \\
\text { desempleados } \\
(\mathbf{2 0 1 0}) \mathbf{- 1 5 - 2 4} \\
\text { anos }\end{array}$ & $\begin{array}{l}\text { Tasa de } \\
\text { empleados } \\
(\mathbf{2 0 1 1}) \mathbf{- 1 5}-24 \\
\text { anos }\end{array}$ & $\begin{array}{l}\text { Tasa de } \\
\text { desempleados } \\
(\mathbf{2 0 1 1}) \mathbf{- 1 5}-\mathbf{2 4} \\
\text { anos }\end{array}$ \\
\hline Brasil & $46.0 \%$ & $18.7 \%$ & $44.6 \%$ & $19.1 \%$ \\
\hline Venezuela & $38.2 \%$ & $14.6 \%$ & $36.9 \%$ & $16.1 \%$ \\
\hline
\end{tabular}

Los números apuntan que tanto en Brasil como en Venezuela los desempleados entre 15 y 24 años son superiores a los números de desempleados con edad superior a 25 años. En el ítem empleados la OIT lleva en consideración aquellos trabajadores que están cubiertos por legislación trabajista y derechos sociales. Esto demuestra que el problema de los jovenes en el cuadro general de desempleo y de pobreza en América Latina de una manera general y en específico en Brasil y en Venezuela es extremamente preocupante y 
complejo, especialmente en situaciones conjuncturales de recesión economica que acaban desembocando en mayores restriciones productivas a los grupos que presentan mayores fragilidades de adaptación a los designios de los mercados.

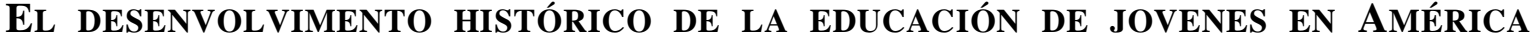 LATINA: COMO ORGANIZAR UN ENSINO MÉDIO PÚBLICO DE CALIDAD PARA TODOS?}

Inicialmente sentimos la necesidad de traer a la luz el que es ser joven en este inmenso continente latino americano, donde la juventud enfrenta las más diversas vicisitudes, los mayores perfiles(marcas) y perjuicios culturales históricos en que ora es vista como problema o como un sector que precisa ser objeto de atención. Entonces Lagree (1999) lanza mano de una reflexión interesante: "controlar la amenza que los segmentos juveniles ofrecen a la sociedad o considerálos como seres en formación amenzados por la sociedad y sus problemas? Es preciso reconocer que, histórica y socialmente, la juventud ha sido considerada como fase de vida marcada por una cierta instabilidad asociada a determinados "problemas sociales y psicológicos" (SPOSITO, 2002).

Veamos que la sociedad ora invirte en las representaciones positivas de los segmentos juveniles, responsables por el cambio social, ora acentuan la dimensión negativa de los "problemas sociales" y del desvío que ser joven representa.

Asi, se en los años 60 la juventud era un "problema" en la medida en que
podia ser definida como protagonista de una crisis de valores y de un
conflicto de generaciones esencialmente situado sobre el ambito de los
comportamentos éticos y culturales, a partir de la década de 1970 los
"problemas" de empleo y de entrada en la vida activa tomaron
progresivamente la delantera en los estudios sobre la juventud, casi
transformando en categoria economica (ABRAMO, 1997, p. 123).

Entre tanto, parte de las atenciones tanto de la sociedad civil como del poder público se volvió, en los últimos años, principalmente para los jovenes y aquellos que están en proceso de exclusión o privados de sus derechos de ciudadanos. Ese doble recorte etario (joven) y economico-social - puede operar con selecciones que acaban por imponer modos propios de concibir las acciones públicas. Se tomadas exclusivamente por la edad cronológica y por los limites de la mayoridad legal, parte de las políticas acaba por excluir un número grande de conjunto de indivíduos que llegan a la mayoridad, pero permanecen en el campo posible de acciones, pues aun viven efectivamente la condición juvenil.

De otra parte, en el conjunto de las imagenes no se considera que, además de los segmentos en proceso de exclusión, hay una inequívoca franjade jovenes pobres, hijos de trabajdores rurales y urbanos (los denominados sectores populares y segmentos oriundos de clases médias urbanas empobrecidas), que hacen parte da larga mayoria juvenil de América Latina y que pueden estar, o no, en horizonte de las acciones públicas, en concecuencia de un modo peculiar de concibilos como sujetos de derechos, pero sin aceso a una condición de vida ciudadana. Según Martins (2010), en la actualidad el ciudadano puede ser visto solamente como el contribuyente, el consumidor; sequeira el princípio constitucional en América Latina de escuela para todos consígue ser cumplido.

Asi, se la educación como instrumento social básico es que posibilita al indivíduo la transposición de la marginalidad para la materialidad de la ciudadania, no es posible, pensar su conquista sin educación. Educar, en esa perspectiva, es entender que derechos humanos y ciudadania 
significan práctica de vida en todas las instancias de convívio social de los indivíduos: en família, en la escuela, en la iglesia, en el conjunto de la sociedad. Sin embargo, basta mirar alrededor para percibir que, en las democracias burguesas, la ciudadania coexiste, contraditoriamente, con las desigualdades. Los derechos son reconocidos como naturales; pero, por las relaciones de poder y explotación, no es asegurado su ejercício ciudadano. (MARTINS, 2010, p. 50-51)

De acuerdo aun con Martins (2010), las políticas educacionales en América Latina son un ejemplo de como el Estado procura producir una aparencia de igualdad de oportunidades y neutralidad, cuando ellas están atadas al movimiento de una economia reglada por el lucro. El Estado surge de una relación entre iguales, como se echase de la voluntad y de los intereses individuales y no de clase, como poder materializado en el derecho y en las instituiciones, constituído por todos los sujetos sociales, sin distinción. Se presentando como elemento neutro, benefactor/protector de las clases sociales, pasa a ser la garantia del trabajador asalariado y no solo la burguesia. "Interponiendo limites negativos o promoviendo el ajuste social, el Estado, objetivando en instituiciones, aparece como encarnación de una racionalidad general y no capitalista" (O’DONNEL, 1981, p. 74).

Asi es posible afirmar que el Estado interfiere directamente en el proceso histórico de formación de la ciudadania popular, por medio de políticas sociales desmovilizadoras y controladoras, generando una sociedad civil que no se reconozca como sujeto de un proyecto emancipatório.

El resultado muestra una paradoja: concepciones igualitárias y universalistas de derechos reactualizan la tradición de una ciudadania restricta, apoyada en la lógica de la expansión de privilégios y no en la universalización de derechos" (TELLES, 1994, p.11). la construcción de la ciudadania y de una cultura basada en los derechos sociales y políticos constituye, hoy, un de los procesos más importantes para la consolidación del proceso de democratización en América Latina.

La relación entre ciudadania y democracia se explicita en el hecho de que ambas son proceso. Los ciudadanos en una democracia, no son solo titulares de derechos ya establecidos, existiendo, en abierto, la posibilidad de expansión, de creación de nuevos derechos, de nuevos espacios, de nuevos mecanismos. El proceso no se dá en un vacio; la ciudadania exige instituiciones, mediaciones y comportamientos propios, se constituyendo en la creación de espacios sociales de lucha y en la definición de instituiciones permanentes para la expresión política. (BENEVIDES, 1998, p. 168)

De esta forma destacamos que la ciudadania requiere la conciencia clara sobre el papel de la educación y las nuevas exigencias empleadas para la escuela que, como instituición para el ensino - la educación formal -, puede ser un lócus excelente para la construcción de la ciudadania. Una escuela autonoma y de calidad, en que el saber transmitido oportunize a todos la capacidad de ejercela con dignidad.

La educación, como práctica efectiva, representa decidida investidura en la construción de la ciudadania. Entre tanto, presenta historicamente carácter restricto, conviviendo con una parte excluída. La igualdad de derechos no solo no suprimió las desigualdades sociales, como las reforzó, al inmascáralas sob el princípio de la libertad. Para que la práctica educativa sea práxis, es preciso que sea hecho en el ambito de un 
proyecto que, además de la intencionalidad, suponga condiciones objetivas de concretización. La escuela es el lugar institucional del proyecto educacional. Se debe establecer como espacio-tiempo, como instancia social mediadora y articuladora del pensar de la juventud. Considerar la formación de la ciudadania como fundamental para consolidación de la democracia subentiende que las instituiciones escolares sean democráticas, que alli haya tolerancia para los diversos segmentos de la sociedad se volviendo principalmente para los jovenes. (MARTINS, 2010, p. 50-51)

Tornarse ciudadano está lejos de agotarse como aquisición legal de un conjunto de derechos, pero se constituye en nuevas formas de sociabilidad en que es imposible ser ciudadano solamente en el discurso, la escuela deve ser un lócus privilegiado del ejercício de la ciudadania para la juventud, en los espacios del cotidiano, en la relación profesoralumno, en el diálogo democrático, en la construción del Proyecto Político Pedagógico de la escuela, en el derecho de adquirir un conocimiento basado en su realidad, en la posibilidad de ser agente ativo de la construción del conoecimiento en sala de aula, en la constituición de un Gremio escolar etc.

La definición y categorización social de la juventud como grupos o agregados de personas que comparten características comunes es algo relactivamente reciente. Se relaciona con el alargamiento de la vida en el período de aquisición de capacidades y habilidades para ingresar en el mundo productivo.

Esto no significa que hablamos hoy sobre una tabula rasa. Lejos de eso, ya hay mucho acumulado en el camino. Jovenes como promesa, transito, amenaza. Confianza y desconfianza en las formas como la juventud recrea la vida social. La juventud, mal entendida como "moratória", o sea, una espécie de limbo necesário, donde se aplaza la construción de vidas propias para, primero, juntase capacidades que rendiron sus frutos en el futuro (lo que más lejos de la juventud de lo que esta idea de parentesis existencial?). Aun más: jovenes como portadores de cambio, con mayor autonomia moral de que los niños, pero sin la autonomia material de los adultos, expuestos a riesgos y provocadores de riesgos, desbordantes en la produción de sentido y mediaciones culturales. Jovenes como objeto de preocupación y sujetos de transformación. (ABRAMO, 1997, p.135)

El imaginário adulto aun muchas veces "vincula la juventud a la ruptura y anomia en la sociedad: debilidade del orden normativo, falta de disciplina en el estudio o trabajo, imprevisibilidad en las reacciones y trayectórias o proliferaciones de conductas de riesgo" (RUA, 1998, p. 744). De acuerdo con Rua (1998), los discursos y instituiciones exaltan y, al mismo tiempo, estigmatizan los jovenes. Es atribuído a los jovenes el papel de protagonista en las modernidades, pero al mismo tiempo son asociados a la violencia, sobretudo si so hombres, urbanos y de estratos populares.

La educación ha sido enfaticamente identificada como factor esencial para lograr el desarrollo integral de los jovenes, algo que podría equalizar gran parte de las expectativas juveniles. En la medida en que el conocimiento científico-tecnológico tornase cada vez más fundamental en los procesos de desenvolvimento economico y social, el papel de la educación adquiere también mayor relevancia. En este sentido para Sola (1998), la educación es un de los principales factores para impulsar el desenvolvimento tanto de los indivíduos como de las comunidades. La auctora aun afirma que una educación básica de calidad desenvolve las capacidades de las personas para que puedan ejercer sus derechos y 
libertades, recrear su cultura, participar de los sistemas de contribuiciones y retribuiciones (sobretodo mediante el trabajo) y obtener asi el bienestar y la protección social.

\begin{abstract}
En una perspectiva social, una buena educación con acceso universal es el principal fundamento para democratizar el desenvolvimento de capacidades y, con eso, el posterior acceso a las oportunidades. Constituye, se regida por los princípios de eqüidade y calidad, el mecanismo más importante de inclusión social en el transito de una generación a la otra. Por otro lado, la mayor inclusión social también es básica para una oferta y demanda más igualitárias de educación. Asi, sociedades con buena educación para todos tiene una estructura de renta más eqüitativa. (SOLA, 1998, p. 96)
\end{abstract}

En consonancia con este cuadro, Gadotti (1994) afirma que la escuela se caracteriza como la instituición de la mediación de la vida real, tornando la institucionalidad efectiva, concreta, histórica, a fin de que los objetivos intencionalizados no queden solo en el plan del ideal, pero sucedan de forma real. Aun afirma el autor que la escuela es el lugar privilegiado para la juventud, pues es en ella que ocurre la intersección del proyecto coletivo de la sociedad con los proyectos existenciales de estes jovenes con los educadores. Entre otros desafios, la escuela deberá construir formas de enfrentamiento para las nuevas exigencias de la sociedad que se anuncia, caracterizada por el avance irresistible y acelerado de la revolución científico-tecnológico, con todas las suyas contradiciones, en un mundo marcado por las desigualdades y sus consecuencias en todos los sectores.

\title{
Conclusiones
}

Algunas cuestiones son importantes en el sentido de entendermos las dificultades encontradas por la escuela para la concretización de su proyecto de transformación ciudadana. En muchos casos las iniciativas de cambio de la escuela son fragilizadas por factores que impeden esa transformación como, por ejemplo, según apunta Gadotti (1994): las formas de gestión, la desconcentración como descentralización, la autonomia outorgada a la escuela sin la devida contrapartida de recursos por parte do Estado, dejando la escuela casi a la deriva delante de una realidad extremamente precária. Es necesario reafirmar la responsabilidad del Estado en la coordinación general de las políticas educacionales que van de hecho garantizar la calidad de ensino y de mantenimiento del sistema como un todo.

Producir políticas públicas de educación de calidad en América Latina volvidas para los jovenes que se utilize de critérios de equiidade y contenidos pertinentes a sus propios contextos sociales y culturales y que sean relevantes para enfrentar con exito y capacidad inovadora los cambios cada vez más constantes en el ambito de toda la sociedad moderna y no solamente para atender las demandas efimeras y productivistas del mercado precarizado es el mayor desafio enfrentado por los gobiernos populares latino americanos.

Para eso, lo que se espera es que la escuela se organize con base en una nueva concepción de conocimiento, operando con teorias de aprendizaje y formas de organización de ensino que superem las prácticas pedagógicas tradicionalmente centradas en la memorización y en la reprodución de informaciones, o en el entrenamiento del saberhacer, ya que la demanda que hoy se propone es por la formación de ciudadanos pensantes, críticos e autónomos. 


\section{Referencias de la literatura}

ARANHA, Maria L. de Arruda. História da educação. São Paulo: Moderna, 1996.

ARCARY, Valério. O encontro da revolução com a história. $1^{\text {a }}$ ed. São Paulo: Xamã Editora, 2006.

BENÍTEZ, Manuel de Puelles. Los sistemas educativos nacionales. Madri: CADE, 2009.

BOMFIM, Manuel - América Latina: males de origem. São Paulo, Editora Paz e Terra, 1993.

BUFFA, Ester. Educação e cidadania burguesa. In. BUFFA, Ester. ARROYO, Miguel G. NOSELLA, Paolo. Educação e cidadania: quem educa o cidadão? São Paulo, Cortez, 2007.

CASALI, Alípio et at. Empregabilidade e educação: novos caminhos no mundo do trabalho. São Paulo: EDUC, 1997.

CASANOVA, R. Venezuela después del Liberalismo: de los consensos de la reforma de los años noventa a un nuevo contrato educativo? (mimeo) 2006.

CARLOT, B. Educação e Globalização: uma tentativa de colocar ordem no debate. Revista de Ciências da Educação, n.4 set/dez. 2007. Disponível em: www.sisifo.fpce.ul.pt. Acesso em 17 de abril de 2009.

CIMADAMORE, Alberto D. et al. Produção de pobreza e desigualdade na América Latina. Organizadores: Antonio David Cattani, Alberto D. Cimadamore ; tradução: Ernani Ssó. — Porto Alegre : Tomo Editorial/Clacso, 2007.

COTRIM, Gilberto. Educação para uma escola democrática. São Paulo: Saraiva, 1991.

CUNHA, Luiz Antônio; GÓES, Moacyr de. O golpe na educação. $11^{\mathrm{a}}$ Ed. Rio de Janeiro: Jorge Zahar Editor, 2002.

FRIGOTTO, G. Educação e a crise do capitalismo real. 4 ed. São Paulo: Cortez, 2000.

FRIGOTTO, Gaudêncio. CIAVATTA, Maria. Educação básica no Brasil na década de 1990: subordinação ativa e consentida à lógica do mercado. Educ. Soc. [online]. 2003, vol.24, n.82, pp. 93-130.

GADOTTI, Moacir. Autonomia como estratégia da qualidade do ensino e a nova organização do trabalho na escola. In: Paixão de Aprender nº 7. Porto Alegre, jun. 1994.

HIRATA, Helena. Os mundos do trabalho: convergência e diversidade num contexto de mudança dos paradigmas produtivos. In: CASALI, Alípio et at. Empregabilidade e educação: novos caminhos no mundo do trabalho. São Paulo: EDUC, 1997.

INSTITUTO BRASILEIRO DE GEOGRAFIA E ESTATÍSTICA - IBGE. Diretoria de Pesquisas, Departamento de População e Indicadores Sociais. Rio de Janeiro, IBGE, 2000.

IANNI, Otávio. Classe e nação. Petrópolis: Vozes, 1986.

JÁUREGUI, Luis Bravo. La educación en tiempos de Chávez. Una revisión crítica y académicamente orientada del proceso de la educación nacional a partir de 1999. Venezuela: El Nacional, 2006.

LUCENA, Carlos. (Org.). Capitalismo, Estado e Educação. Campinas, SP: Editora Alínea, 2008. 
MARX, K. \& ENGELS, F. Manifesto do Partido Comunista. São Paulo: Boitempo, 1998.

PRADO, Maria Lígia - O Populismo na América Latina. São Paulo, Editora Brasiliense, 1981 - Coleção Tudo é História.

RIBEIRO, Darcy. Encontros com a civilização brasileira. Rio de Janeiro, Civilização Brasileira, 1978.

SADER, Emir. Latinoamericana: Enciclopédia Contemporânea da América Latina. São Paulo, Boitempo Editorial, 2006.

SAVIANI. D. Tendências e correntes da educação brasileira. MENDES, D. T. (Coord.) Filosofia da Educação Brasileira. 3 ed. Rio de Janeiro: Civilização Brasileira, 1987.

WASSERMAN, Claudia (coordenação) - História da América Latina: Cinco Séculos. Porto Alegre, Editora da Universidade, 2000.

\section{Notas}

\footnotetext{
${ }^{1}$ Professor da Secretaria de Estado de Educação do Distrito Federal e atualmente professor no Departamento de Educação Física da Universidade de Brasília.

${ }^{2}$ Por la definición de las Naciones Unidas, utilizada también por la OIT (Organización Internacional del Trabajo),l os jovenes son el grupo etario entre los 15 y 24 anos, pero varia de país para país. En los países industrializados, la edad mínima limite corresponde, en general, a la edad mínima del fin de la escolaridad obligatória. La máxima varia. En los países en desenvolvimento, la edad mínima limite también varia. En América Latina y los países del Caribe las estadísticas oficiales de población economicamente activa tiene los 10 años de edad por base, mismo que esa edad sea o se haya tornado ilegal para trabajar.

${ }^{3} \mathrm{El}$ informe la OIT denominado "Trabajo decente y Juventude: América Latina" trata de las condiciones de trabajo y estudio de los jovenes latino americanos entre 15 y 24 años. Oficina Regional para América Latina y Caribe, 2011, con base en tabulaciones especiales de las investigaciones domiciliares.
}

Recebido em fevereiro-2013

Aprovado em outubro-2013 